\title{
Light-addressable amperometric electrodes for enzyme sensors based on direct quantum dot-electrode contacts
}

Riedel, M., Göbel, G., Parak, W., Lisdat, F.

M. Riedel, G. Göbel, W. J. Parak, F. Lisdat, "Light-addressable amperometric electrodes for enzyme sensors based on direct quantum dot-electrode contacts," Proc. SPIE 8955, Colloidal Nanoparticles for Biomedical Applications IX, 89551M (24 March 2014); doi: 10.1117/12.2044601 


\title{
Light-addressable amperometric electrodes for enzyme sensors based on direct quantum dot-electrode contacts
}

\author{
M. Riedel ${ }^{\text {a }}$, G. Göbel ${ }^{\text {a }}$ W. J. Parak ${ }^{\text {b }}$ F. Lisdat*a \\ ${ }^{a}$ Biosystems Technology, University of Applied Science Wildau, Bahnhofstrasse 1, 15745 Wildau, \\ Germany; biophotonics, Philipps University Marburg, Renthof 7, 35037 Marburg, Germany
}

\begin{abstract}
Quantum dots allow the generation of charge carriers upon illumination. When these particles are attached to an electrode a photocurrent can be generated. This allows their use as a light-switchable layer on the surface. The QDs can not only exchange electrons with the electrode, but can also interact with donor or acceptor compounds in solution providing access to the construction of signal chains starting from an analyte molecule. The magnitude and the direction of the photocurrent depend on several factors such as electrode polarization, solution $\mathrm{pH}$ and composition. These defined dependencies have been evaluated with respect to the combination of QD-electrodes with enzyme reactions for sensorial purpose. CdSe/ZnS-QD-modified electrodes can be used to follow enzymatic reactions in solution based on the oxygen sensitivity. In order to develop a photoelectrochemical biosensor, e.g. glucose oxidase is immobilized on the CdSe/ZnSelectrode. One immobilization strategy applies the layer-by-layer-technique of GOD and a polyelectrolyte. Photocurrent measurements of such a sensor show a clear concentration dependent behaviour. The principle of combining QD electrodes with a layered architecture and light triggered read-out can also be transferred to other enzymes such sarcosine oxidase. The sensitivity of quantum dot electrodes can be influenced by additional nanoparticles, but also by multiple layers of the QDs. In another direction of research it can be demonstrated that direct electron transfer from excited quantum dots can be achieved with the redox protein cytochrome c. This allows the detection of the protein, but also interaction partners such as enzymes or superoxide.
\end{abstract}

Keywords: photo electrochemistry, quantum dots, enzymes, photocurrent, sensor

\section{INTRODUCTION}

Quantum Dots (QDs) are semiconductor nanoparticles which have been widely used in various disciplines because of their unique optical properties. [1,2] For the synthesis mainly II-IV-Semicondutor (e.g. CdSe, CdS, CdTe, ZnS) are used. To increase the quantum yield and the stability towards photobleaching effects QDs are often covered with a second semiconductor according to the core-shell-principle (e.g. CdSe/ZnS). Due to the semiconductor properties of QDs electron hole pairs can be generated inside the particle upon illumination with a proper wavelength. Thereby an electron switches from the valence band in the conduction band and leaving a defect electron in the valence band. The relaxation of the excited electron in the initial state results in light emission, depending on the size of the QD. With increasing diameter the wavelength of the emitted photons is shifted to higher values, allowing a size-tuneable fluorescence. Because of the optical properties QDs have become very popular fluorescence labels in bioanalytical applications allowing highly parallel and multiplexed analysis.

In addition to the use of QDs as optical marker, combinations of QDs with electrodes as new electrochemical building blocks have been discussed in several studies [3-10]. While without illumination, QDs immobilisied on electrodes act as a rather insulating layer and hindering electrochemical conversions, introducing light to the system enables a electron transfer reaction between QDs and the electrode. When electron-hole-pairs are generated inside the nanoparticles, electrons can be removed or introduced by the electrode, resulting in the generation of a photocurrent which can be used as analytical tool. This provides the basis for the use of QDs as photo-sensitive layer for the construction of lighttriggered sensors. Here, the magnitude of the current is affected by the applied potential and the presence of an electroactive substrate which can act as a electron acceptor and donor. As shown in figure 1 a cathodic or anodic photocurrent 
can be triggered depending on the applied potential. Cathodic photocurrents caused by electron transfer reactions from the electrode via the QDs to a acceptor in solution. On the other hand the electron flow from a donor in solution via the QD to the electrode can occur by generating an anodic photocurrent. For a effective photocurrent generation different factors such as the quality of the QDs, the coupling of QDs to the electrode, the reaction rates for oxidation or reduction of substances at the QDs and the recombination of charge carriers inside the nanoparticles possess an important impact [11].

Various procedures have been developed to immobilize QDs on electrodes and improve the biocompatibility of QDsurfaces for bioanalytical applications. Typically QDs are synthesized by chemical reactions in organic solvents and have been covered with stabilizing nonpolar ligands such as trioctylphosphine oxide (TOPO). By ligand exchange, the nonpolar ligand of the synthesized QDs can be replaced by ligands with functional groups to provide functionalities for electrode and/or biomolecule interaction. Here, dithiol and disulfide compounds (e.g. 1,6-hexanedithiol, 1,4dithiobenzene, 1,4-dithiane) are often used to convey a connection between gold electrodes and QDs via chemisorptions [4]. Another strategy to change the surface properties of the QDs based on the coating with different coupling agents such as polymers [12]. The introduced functionalities can be used for further modification steps like the direct coupling with biomolecules through covalent connection, bioaffinity binding or electrostatic interactions. By electrostatic interactions multilayer-systems not only with biomolecules can be realized, but also layered architectures with QDs have been shown to increase the photocurrents [10].

Here, some light-triggered biosensor systems based on the combination of QDs and enzymes will be presented. Different operation principles have been used for the sensors in order to generate photocurrent changes in the presence of the analyte: Detection of the formation of an electro-active enzymatic product, monitoring the consumption of co-substrate or applying direct QD-enzyme interactions (direct protein electrochemistry)..
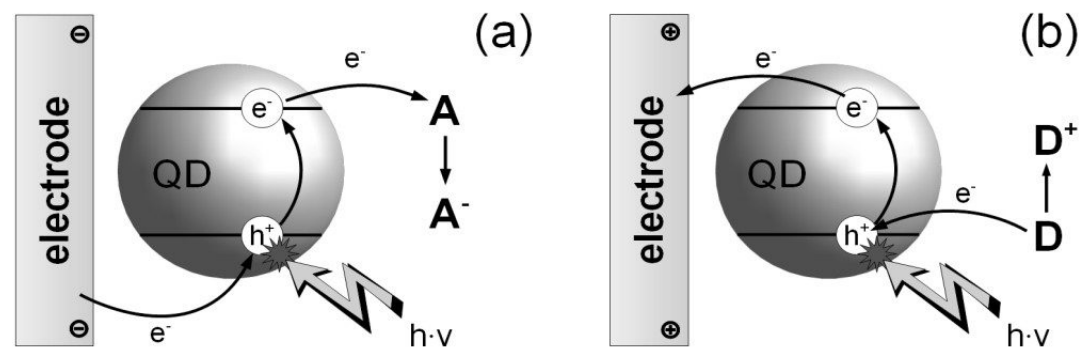

Figure 1. Illustration of the electron transfer steps at a quantum dot modified electrode after illumination. (a) cathodic cascade an (b) anodic cascade. " $\mathrm{A}$ " and " $\mathrm{D}$ " represents the acceptor and donor, respectively.

\section{NADH-DEPENDENT DEHYDROGENASE REACTIONS}

In this study we want to investigate the applicability of QD modified electrodes for the sensorial detection of NADH [3]. Therefore, CdSe/ZnS QDs have been immobilized on a gold electrode by a dithiol compound to provide a photoswitchable layer. After addition of NADH a change of the photocurrent can be observed in a rather wide potential range around $0 \mathrm{~V}$ vs. $\mathrm{Ag} / \mathrm{AgCl}$. At positive potentials where an anodic photocurrent can be detected, the signal enhances in the presence of NADH, which indicates that electrons transferred from NADH to the excited QDs under illumination. However, at potentials which induce a cathodic photocurrent, this current decreases after addition of NADH. Obviously the electrons transferred from the electrode to the excited QDs compete with electrons from NADH. At an appropriate potential a cathodic photocurrent can be transformed into an anodic photocurrent in the presence of NADH. Thus, the direction of the photocurrent can be reversed by a chemical reaction. The magnitude of photocurrent change due to the $\mathrm{NADH}$ oxidation is in the potential range from -100 to $+200 \mathrm{mV}$ vs. $\mathrm{Ag} / \mathrm{AgCl}$ similar and shows that NADH oxidation cannot simply be increased by the applied bias. To avoid large background signals in the absence of the analyte the sensitivity of the QD electrode for $\mathrm{NADH}$ has been investigated at a potential of $+50 \mathrm{mV} v \mathrm{vs} . \mathrm{Ag} / \mathrm{AgCl}$ and is found that 
the electrode exhibits a range of detection between $2 \mu \mathrm{M}$ and $2 \mathrm{mM}$. This provides the basis for the combination of QDs with dehydrogenase reactions for the detection of substrates by means of the cofactor NADH.

To check the feasibility of the concept glucose dehydrogenase (Pseudomonas sp.) has been chosen as biocatalyst. The enzyme catalyzes the oxidation of glucose to glucono-1,5-lactone by the reduction of NAD+ to NADH. While photocurrent measurements with QD modified electrodes in the presence of glucose without glucose dehydrogenase show no photocurrent change at low potentials, the addition of the enzyme and NAD + evokes a substrate concentration dependent change of the signal. Because glucose cannot be oxidized directly at the QD electrode the photocurrent change can be attributed to the conversion of the glucose signal to NADH by electron transfer via the enzyme, followed by a detection of NADH by electron transfer to the illuminated QDs. The determined concentration range for glucose is relatively similar to that of NADH. This system demonstrates that QD modified electrodes in combination with a biocatalyst can be used for the construction of bioanalytical signal chains enabling light-triggered analysis of substrates of NADH converting enzymes.

\section{DIRECT PROTEINELECTROCHEMISTRY AT QD-ELECTRODES}

Another aspect of research which has been investigated is the direct protein electrochemistry at QD modified electrodes. This is aimed to establish direct heterogeneous electron transfer (DET) between the redox centre of the protein and the QD electrode. We have found that the surface properties are essential for the observation of a DET with a protein. While organic capping agents (e.g. trioctylphosphine oxide) on the QD surface prevent the electron transfer with cytochrome c, the exchange of the hydrophobic surfactant to a hydrophilic modifier (e.g. mercaptopropionic acid or mercaptosuccinic acid) makes electron transfer reactions feasible [5]. Thus, DET between a redox protein (cytochrome c) and illuminated QDs has been observed, allowing the construction of analytical signal chains for the detection of analyte molecules in solution.

In a first approach the QD electrode have been combined with cytochrome $\mathrm{c}$ for the establishment of a photoelectrochemical signal chain which is sensitive for superoxide radicals [6]. For the measurement a potential of $+130 \mathrm{mV}$ (vs Ag/ $\mathrm{AgCl}$ ) is applied to ensure that only reduced cytochrome $\mathrm{c}$ is detectable by photocurrent changes. When superoxide radicals are generated in solution via xanthine oxidase catalysed conversion of hypoxanthine to uric acid in the presence of oxidized cytochrome $\mathrm{c}$ a increasing photocurrent can be observed. This means that produced superoxide radicals reduce the oxidized cytochrome $\mathrm{c}$ in solution. Due to the excitation of the QDs by illumination the cytochome $\mathrm{c}$ is re-oxidized at the electrode and enhances the photocurrent. Thereby the magnitude of photocurrent enhancement correlates with the superoxide radical concentration and enables the detection in the nanomolar range.

Another approach based on cytochrome c mediated biocatalytic cascades. Here, for example the combination of QD electrodes with cytochrome $\mathrm{c}$ and lactate dehydrogenase (LDH) or nitrate reductase (NR) has been used for the amplification of the photocurrent in presence of their respective substrates [7]. The anodic photocurrent increases in the presence of $\mathrm{LDH}$ and lactate with rising substrate concentration up to saturation at a $70 \mathrm{mM}$. Thereby the photocurrent is approximately 9-fold higher than the value observed with reduced cytochrome c only. In comparison an increasing cathodic photocurrent can be observed when NR is used and increasing nitrate concentrations are added. The saturated photocurrent can be amplified up to approximately 8 -fold.

\section{COMBINATION OF OXYGEN CONSUMING REACTIONS WITH QDS}

\section{Effect of oxygen on the photocurrent}

For the investigation of the influence of oxygen on the cathodic photocurrent, after immobilization of CdSe/ZnS QDs at a gold electrode by a dithiol, the QD-electrode is analyzed in air-saturated and argon-purged buffer. Measurements in air-saturated buffer show a higher photocurrent compared to measurements in argon-purged buffer indicating that a part of the signal depends on the oxygen in the solution. Here, the oxygen dependent photocurrent is influenced by the $\mathrm{pH}$ and the applied potential. At potentials less then $-0,3 \mathrm{~V}$ vs. $\mathrm{Ag} / \mathrm{AgCl}$ and a neutral $\mathrm{pH}$ maximum photocurrent generation can be ensured. Here the oxygen-dependent part of the photocurrent is around $50-60 \%$. This means that oxygen serve 
as an electron acceptor at the illuminated QDs and prevents a complete recombination of the electron hole pairs inside the QDs, resulting in a constant photocurrent which proves to depend linearly on the oxygen concentration in solution.

The observed oxygen dependence provides the basis for a sensorial application by coupling of the QD-electrode with a oxidase to detect the substrate of the enzyme reaction. As illustrated in figure 2, in the presence of substrate the enzyme reaction leads to a consumption of the co-substrate oxygen, which is lacking for the reduction at the QDs: So a competitive situation between the oxidase and the QDs with respect to oxygen is obtained, resulting in a signal change.

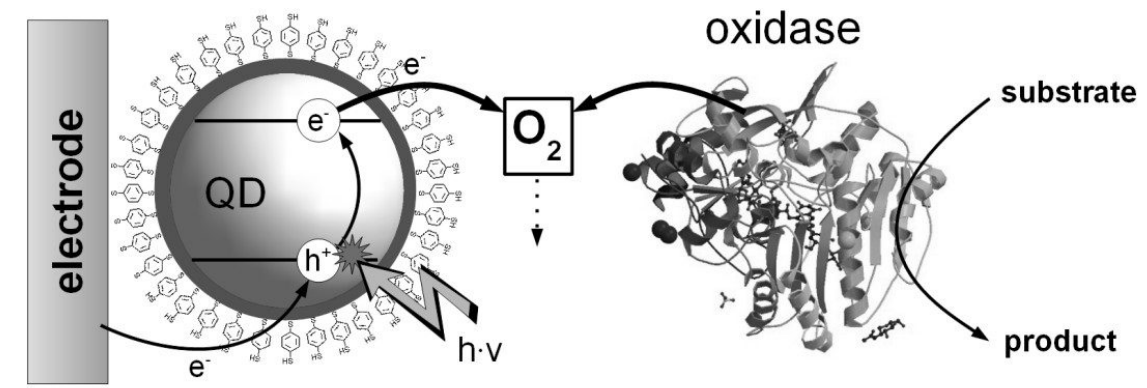

Figure 2. Sensor principle of a QD-electrode in combination with a oxidase for the photoelectrochemical substrate detection. The light-excited QDs and the oxidase compete for the available oxygen in solution.

\section{Investigations of Oxidase-QD-electrodes}

In order to demonstrate that the principle works first glucose oxidase (GOD) has been chosen for the combination with QDs [8]. The analysis of GOD in solution leads to a suppression of the photocurrent when the substrate is present, allowing the determination of GOD activities down to $0.025 \mathrm{U} / \mathrm{ml}$. Furthermore, GOD can be fixed to a QD-electrode by different immobilization strategies with the aim to attach a high enzyme density. This is necessary to achieve well defined oxygen depletion in front of the QD surface. In one approach, chemical crosslinking with the bifunctional reagent glutaraldehyde is used. Another strategy uses the layer-by-layer assembly with the polyelectrolyte poly(allylamine hydrochloride) (PAH). This is based on the electrostatic interactions between the negatively charged enzyme and the positively charged polyelectrolyte at $\mathrm{pH}$ 7. This method enables not only the preparation of defined structures, but also a better control of the attached enzyme density. By means of QCM, conditions for the successful construction of a multilayer system can be evaluated. The alternating assembly of GOD and PAH in 2 mM sodium phosphate buffer $\mathrm{pH} 7$ has been followed up to four bilayers. Thereby the amount of the deposited GOD is rather constant for the different adsorption steps $(\Delta \mathrm{f}=77 \pm 10 \mathrm{~Hz})$.

The attachment of GOD to the QD electrode leads in the presence of glucose to a photocurrent suppression depending on the substrate concentration in solution. The sensitivity of the sensor can be adjusted by the concentration of the attached enzyme through the variation of the number of [GOD/PAH]n-layer on QD electrodes. As illustrated in Figure 3, all multilayer systems in the range of $2-6$ layers show photocurrent suppression in the presence of substrate. The magnitude of signal becomes more enhanced as the number of layers increases (up to four layers). The six layer system does not further increase the sensitivity in the dynamic range between $100 \mu \mathrm{M}$ and $5 \mathrm{mM}$ glucose. This means that glucose can diffuse in the whole layer system (up to four layers) and enzyme molecules near to the electrode are also contributing to the generation of the sensor signal. Under substrate saturation up to $50 \%$ of the photocurrent can be suppressed. This corresponds to the whole oxygen depended part of the photocurrent. Therefore it can be concluded that the four layer system already uses the complete oxygen sensitivity of the QD electrode. After substrate measurement, the sensor can be regenerated in buffer solution and is ready for further analysis. The system shows the ability for a sensitive detection of glucose in the range of $100 \mu \mathrm{M}$ and $5 \mathrm{mM}$ and could be beneficial for the determination of glucose concentrations in blood or plasma $(3-11 \mathrm{mM})$ when the sample will be diluted. 
Photocurrent measurements of QD electrodes without enzymes in glucose solution do not evoke a signal change. Thus a direct conversion at the illuminated QDs can be excluded. Furthermore, some potentially disturbing molecules, such as hydrogen peroxide, urea and glycine are studied. No influence on the photocurrent behavior is found when these molecules added under working conditions with concentrations of $200 \mu \mathrm{M}$.

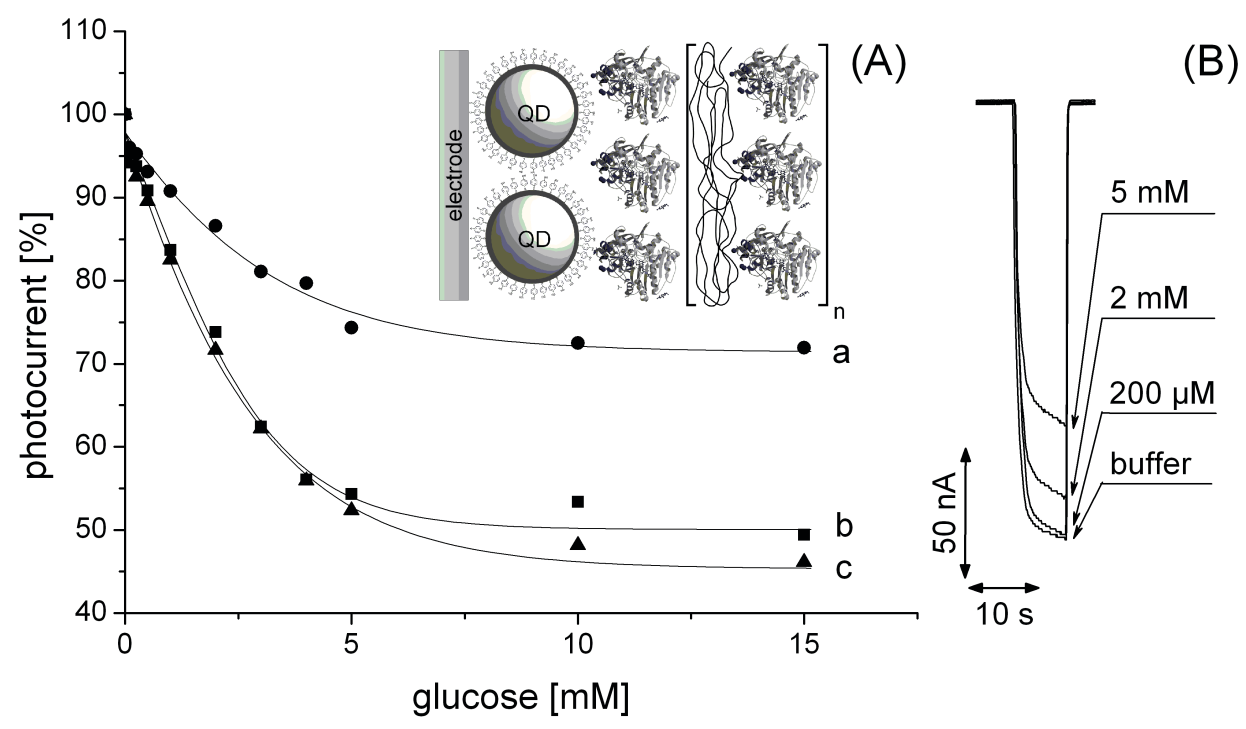

Figure 3. Relative change of photocurrent of different QD modified electrodes with immobilized $[\mathrm{GOD} / \mathrm{PAH}]_{\mathrm{n}}$ layer depending on the glucose concentration in the range of $0-20 \mathrm{mM}$; a) $[\mathrm{GOD} / \mathrm{PAH}]_{2}$, b) $\left.[\mathrm{GOD} / \mathrm{PAH}]_{4}, \mathrm{c}\right)[\mathrm{GOD} / \mathrm{PAH}]_{6}$ (100 mM HEPES pH 6,8; illumination time $t_{\mathrm{L}}=10 \mathrm{~s}, \mathrm{E}=-350 \mathrm{mV}$ vs $\mathrm{Ag} / \mathrm{AgCl}$ ). Inset (A) Schematic illustration of the $[\mathrm{GOD} / \mathrm{PAH}]_{\mathrm{n}}$-layer assembly on the QD electrode. Inset (B) Photocurrent measurement of an QD electrode with a $[\mathrm{GOD} / \mathrm{PAH}]_{2}$-layer system in solutions with different glucose concentrations.

In an analogous system with the enzyme sarcosine oxidase (SOD) the amino acid sarcosine have been detected [9]. Thereby, also a layer-by-layer assembly with the polyelectrolyte PAH has been used to attach the enzyme to the QD electrode and thus the sensor can measure sarcosine with a linear range from $100 \mu \mathrm{M}$ to $1 \mathrm{mM}$.

This sensor principle has great potential for a parallel analysis of different enzyme substrates when the corresponding biocatalyst is separately fixed on the QD electrode surface and a spatially resolved illumination is used. However, the spatially resolved immobilization of the enzyme in small spots needs further investigations.

\section{CONCLUSIONS}

In this work, various photobioelectrochemical sensor principles have been demonstrated based on QD modified electrodes. It is shown that QDs are useful building blocks on electrodes which enable a light-directed read-out. By shining light onto the system electron-hole pairs are generated in the QDs enabling electron transfer reactions between the QDs and the electrode, but also between QDs and donor or acceptor compounds in solution. Firstly the determination of NADH concentrations at a QD electrode can be shown at low potentials, which allows the detection of substrates of enzymes which show participation of NADH in the cycle. Additionally, a direct interaction of excited QDs and cytochrome $\mathrm{c}$ after surface modification is demonstrated opening the way to the analysis of enzyme systems capable to react with cyt $\mathrm{c}$. Another approach uses the oxygen dependence of the cathodic photocurrent of $\mathrm{CdSe} / \mathrm{ZnS}-\mathrm{QD}$ electrodes. This provides the basis for the detection of oxygen consuming reactions and has been demonstrated with glucose oxidase and sarcosine oxidase. 


\section{REFERENCES}

[1] Parak, W. J., Pellegrino, T. and Plank, C., "Labelling of cells with quantum dots", Nanotech., 16, R5-R25, (2005).

[2] Wang, C., Gao, X. and Su, X. G., "In vitro and in vivo imaging with quantum dots", Anal. Bioanal. Chem., 397 , 1397-1415, (2011).

[3] Liu, Q., Lu, X.B., Li, J., Yao, X. and Li, H., "Direct electrochemistry of glucose oxidase and electrochemical biosensing on quantum dots/carbon nanotubes electrodes", Biosens. Bioelectron., 22, 3203-3209, (2007).

[4] Schubert, K., Khalid, W., Yue, Z., Parak, W.J. and Lisdat, F., "Quantum-Dot-Modified Electrode in Combination with NADH-Dependent Dehydrogenase Reactions for Substrate Analysis", Langmuir, 26, 13951400, (2010).

[5] Stoll, C., Kudera, S., Parak, W.J. and Lisdat, F., "Quantum Dots on Gold: Electrodes For Photoswitchable Cytochrome c Electrochemistry", SMALL, 2, 741-743, (2006).

[6] Stoll, C., Gehring, C., Schubert, K., Zanella, M., Parak, W.J. and Lisdat, F., "Photoelectrochemical signal chain based on quantum dots on gold - Sensitive to superoxide radicals in solution", Biosens. Bioelectron., 24, 260265, (2008).

[7] Katz, E., Zayats, M., Willner, I. and Lisdat, F., "Controlling the direction of photocurrents by means of CdS nanoparticles and cytochrome c-mediated biocatalytic cascades", Chem. Commun., 1395-1397, (2006).

[8] Tanne, J., Schäfer, D., Khalid, W., Parak, W.J. and Lisdat, F., "Light-Controlled Bioelectrochemical Sensor Based on CdSe/ZnS Quantum Dots“, Anal. Chem., 83, 7778-7785, (2011).

[9] Riedel, M., Göbel, G., Abdelmonem, A.M., Parak, W.J. and Lisdat, F., "Photoelectrochemical Sensor Based on Quantum Dots and Sarcosine Oxidase", ChemPhysChem, 14, 2338-2342, (2012).

[10] Göbel, G., Schubert, K., Schubert, I.W., Khalid, W., Parak, W.J. and Lisdat, F., "Enhanced photocurrent generation with quantum dots containing multilayers on gold", Electrochim. Acta, 56, 6397-6400, (2011).

[11] Lisdat, F., Schäfer, D. and Kapp, A., "Quantum dots on electrodes - new tools for bioelectroanalysis“, Anal. Bioanal. Chem., 405, 3739-3752, (2013).

[12]Fogg, D.E., Radzilowski, L.H., Dabbousi, B.O., Schrock, R.R., Thomas, E.L. and Bawendi, M.G., "Fabrication of Quantum Dot-Polymer Composites: Semiconductor Nanoclusters in Dual-Function Polymer Matrices with Electron-transporting and Cluster-Passivating Properties, Macromolecules, 30, 8433-8439, (1997). 